\title{
ORDERING IN MONOLAYER HELIUM FILMS ON GRAPHITE
}

M. Bretz

Dept. of Physics, University of Michigan, Ann Arbor, Mich., U.S.A.

Résumé.- Nous discutons des configurations de couches epitaxiques mono-atomiques physiquement réalisables dans 1 'optique du modèle de Potts à q états. Nous présentons, le diagrame de phase de l'hélium 2D et une analyse critique de la phase ordonnée. Nous présentons aussi des prévisions théoriques sur la transition ordre-désordre et discutons les possibilités d'expériences futures.

Abstract.- Some physically realizable epitaxial monolayer configurations and their relationship to the q-state Potts Model are discussed. The phase diagram for $2 \mathrm{D}$ helium is surveyed and a critical analysis of the ordered phase is described. Theoretical expectations for the order-disorder transition and possible future experimentation are presented.

There is currently considerable activity in ordered monolayers adsorbed on basal plane graphite /1/. A wide variety of adsorbates (noble gases, molecules, hydrocarbons) order in several 2 dimensional epitaxial configurations, some of which correspond to important theoretical models $/ 2 /$. Phase diagrams for many of these systems have been mapped by careful vapor pressure measurements $/ 3 /$ and the implied molecular configurations confirmed by Leed and neutron scattering studies $/ 4 /$. Heat capacity and NMR probes have in some cases explored the thermodynamic and dynamic film properties respectively /4/. The pertinent commensurate systems for our discussion are shown in figure 1. The graphite surface presents a mosaic of potential wells which adsorbates seek out. At appropriate temperatures and densities ordered solids are formed with the adatom crystals constrained to the periodicity of the underlying graphite. At the top of the figure two such monolayer solids are schematically shown. Since the hard cores are larger than the graphite hexagonal wells $(5.24 \AA)^{2}$ occupancy of nearest neighbor wells is prohibited resulting in an adatom filling of $1 / 4$ or $1 / 3$ of the possible potential sites. One can think of 4 or 3 equivalent sublattices respectively where crystals can nucleate on the infinite graphite plane. As a $2^{\text {nd }}$ order phase transition is approached form high $T$, crystal clusters on different sublattices crowd each other and compete for dominance. Grain boundary activity determines the critical properties of the particular system. These clusters can be thought of as distinct macro-particles which attract each other. if of like kind, but do not interact otherwise. The spin system analogy is the extended Lenz-Ising, or q-state Potts mode1 /5/. The Potts mode1 allows $q$ spin orientations on a square lattice with attractive interactions for aligned neighbor spins but no interaction otherwise. The three (four) types of ordered adatom clusters then correspond to three (four) possible spin orientations. The square vrs. triangular array is unimportant close to the phase transition where these microscopic details "renormalize" away. Thus, the $1 / 4$ array (designated $(2 \times 2)$ ) is equivalent to the 4-state Potts model and the $1 / 3$ film (designated $(\sqrt{3} \times \sqrt{3}) \mathrm{R} 30$ ) was shown by Alexander $16 /$ to be isomorphic with the 3-state Potts model. A similar situation occurs for $2^{\text {nd }}$ layer atoms adsorbed on top of a close-packed monolayer (bottom of figure 1). The most favorable potential sites for overlayer adsorption correspond to the vertices of a mosaic with a monolayer atom at the center of each hexagon. If one choses the overlayer adatom hard core diameter to be smaller than the monolayer hard core, then one half of the potential sites can be filled. This correspond to the 2-state Potts or Ising model and should give a logarithmic divergence in the heat capacity when passing through the orderdisorder transition. Alternately, if a large overlayer adatom is chosen, the 4-state Potts model might again be realized.

Physically, many ordered simple monolayer systems seek the $(\sqrt{3} \times \sqrt{3})$ R30 configuration. Krypton, $\mathrm{CH}_{4}$, Xe and $\mathrm{N}_{2}$ layers for example, condense from the 2D liquid by $1^{\text {st }}$ order transitions. A two phase region exists as the monolayer builds. When the surface becomes covered by the epitaxial solid, further addition of adatoms forces crystalline misfit which incites an order-disorder transition. For krypton 


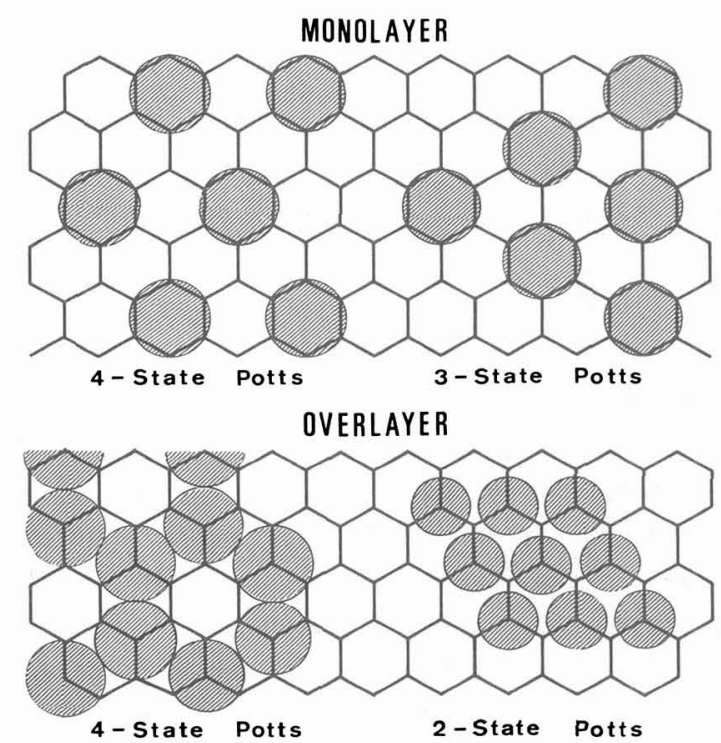

Fig. 1 : Top - Graphite mosaic occupied by adsorbed atoms in two possible epitaxial configurations. Bottom - 2nd layer adatom crystals on a close-packed monolayer

it is thought to be continuous but for other noble gases the nature of the transition is still in question $/ 7 /$. It has been suggested that if one deposits 1/2 layer of $\mathrm{N}_{2}$ on top of the krypton monolayer, the 4-state Potts system might be realized. Conversely, adding 1/2 layer of smaller ${ }^{4} \mathrm{He}$ atoms to the $\mathrm{Kr}$ monolayer should give a lattice gas analogy of the Ising system $/ 8 /$. (Actually, the $\mathrm{N}_{2} / \mathrm{Kr}$ system might prefer to distort in some more complicated manner than inferred from the simple visualization of $\mathrm{fi}-$ gure $1 / 9 /)$.

As an orientation to helium films I present in figure 2 a survey of the monolayer specific heat on Grafoil $/ 10 /$. One can see many distinct regimes as coverage and tempeature are varied.: At the lowest coverages and highest temperatures the film behaves like a non-ideal 2D gas with a specific heat close to 1 . But as $T$ is lowered, the film is thought to condense to a liquid phase marked by the low anoma$1 \mathrm{y}$ near $1.5 \mathrm{~K}$. For highly compressed films near one monolayer there exists a ridge in the specific heat. This is the melting line of the $2 \mathrm{D}$ solid monolayer. $\mathrm{T}^{2}$ behavior below the transitions reveal Debye temperatures quite similar to those at comparable 3D solid helium densities. A full description of these phases is readily available for the interested rea$\operatorname{der} / 12 /$.

The sharp peak near 0.6 monolayer and $3 \mathrm{~K}$ is the 2D gas-ordered solid transition. Both isotopes show essentially identical behavior ruling out mass,
HEAT CAPACITY OF SUBMONOLAYER

$\mathrm{He}^{4}$ FILMS ADSORBED ON GRAPHIT

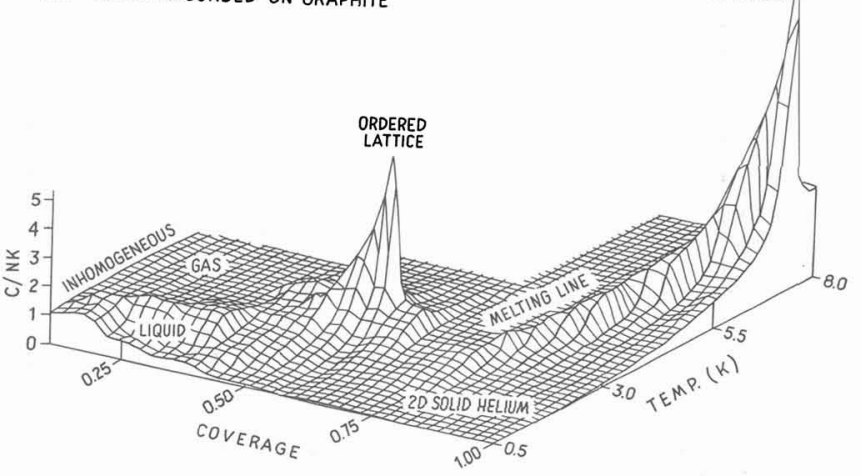

Fig. 2 : Survey of the ${ }^{4} \mathrm{He}$ phase diagram giving specific heat vs. monolayer coverage and temperature

statistics and zero point energy as sensitive variables. (The only difference between the helium ordering transition and those of other noble gases is the appreciable zero point energy and sma11 interparticle interaction). The associated high mobility prevents epitaxial crystal nucleation except over a narrow density range. It was originally thought that the ordering transition was Ising-like since the specific heat for helium on grafoil appeared 1ogarithmic $/ 10 /$. In fact, rouding of the peak due to the small microcrystalline sizes in Grafoil gave an insufficient range in reduced temperature for a good power law fit. Recent1y, I carefully remeasured the specific heat of ordered ${ }^{3} \mathrm{He}$ and ${ }^{4} \mathrm{He}$ using an improved graphite substrate UCAR-ZYX which is exfoliated from a single stress-anealled pyrolytic graphic crystal $/ 11,12 /$. The results for ZYX and Grafoil at critical density are compared in figure 3 . The height of the ZYX peak is twice that for Grafoil and a definite shift in the transition temperature $T_{c}$ of about $20 \mathrm{mK}$ has occurred. Both of these features are consistent with an improvement in micro crystallite size from $\simeq(100 \AA)^{2}$ to $\simeq(350 \AA)^{2}$ or $\simeq x 10$ in effective area.

This new data is amenable to a critical parameter analysis as illustrated in figure 4. Here, the specific heat (with background subtraction) both above and below $\mathrm{T}_{c}$ is plotted vs reduced temperature $t=\left(T-T_{c}\right) / T_{c} \cdot$ A straight line fit corresponds to a logarithmic peak with $\mathrm{C}^{ \pm}=\mathrm{A}^{ \pm}|\mathrm{t}|^{-\alpha}+\mathrm{B}^{ \pm}+\mathrm{Ct}$ and $\alpha=0$ ( \pm refers to $\gtrless T_{c}$ ) One can see the strong upward curvature of the data, however, indicating a large $\alpha$ value. A multiparameter least square fit with $B^{+}=B^{-}$(no discontinuity) results in $\alpha=$ $+0.36 \pm .03$, which is considerably above the 


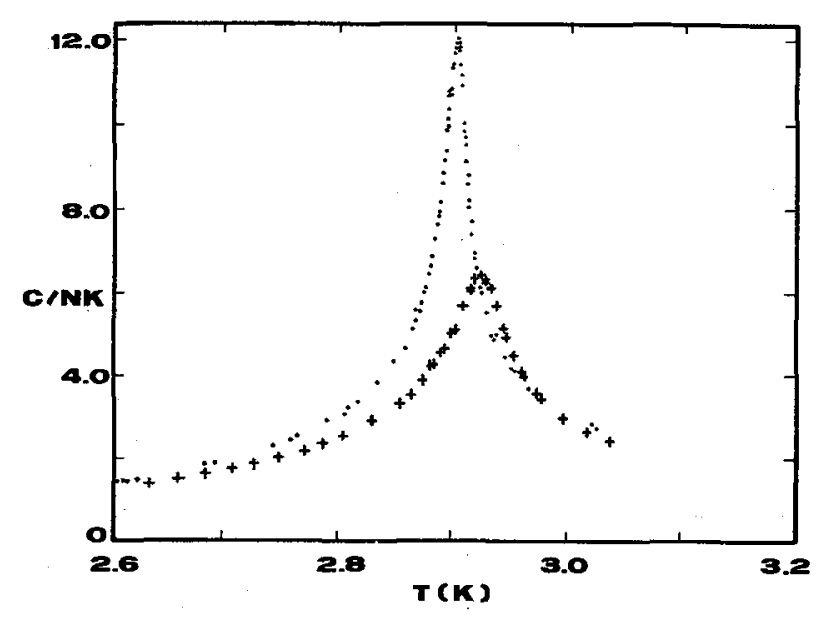

Fig. 3 : Comparison of the helium ordering peak for films adsorbed on ZYX (.) and Grafoil (+)

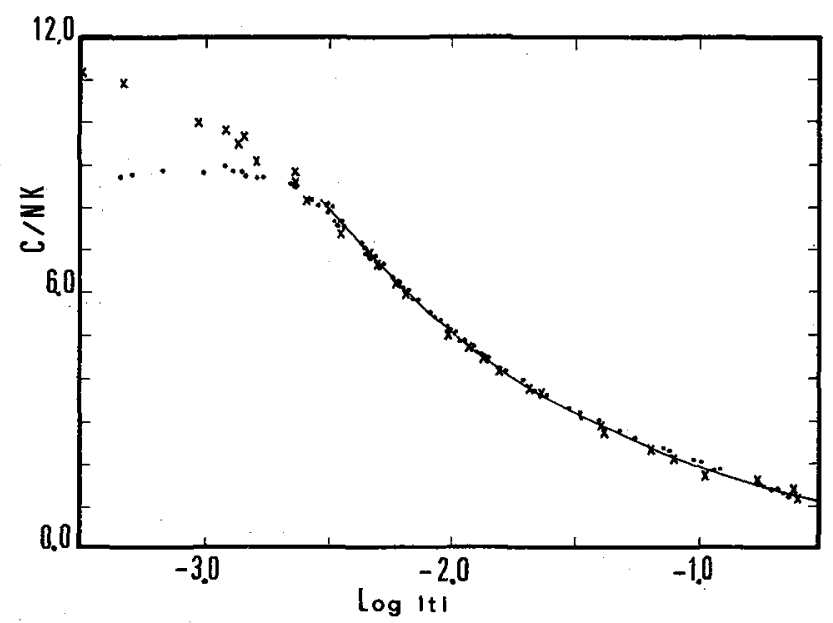

Fig. 4 : Specific heat vs. reduced temperature. The solid line represents the best fit to the data with $\alpha=.36$

$-0.1 \leqslant \alpha \leqslant 0.2$ expected for critical transitions. It appears that the ordering transition in helium films and other $\sqrt{3}$ structures belong, with the 3state Potts model, to a unique universality class not seen previously !

Although Pott's initiated the theoretical

work a few decades ago $/ 5 /$, serious calculations have only recently been attempted. Straley and Fisher $114 /$ using a low temperature series expansion concluded that the 3-state Potts Model on a square 1attice is a higher order transition and Baxter /15/ extended this deduction to the 4-state Potts model. Series expansion values on $\alpha$ now range from 0.05 to $0.26 / 14-16 /$. Recent renormalization group calculations $/ 17 /$ find $\alpha=0.19$ to 0.33 . Theorists de Neef and Enting /18/ have just finished a 22 term series expansion in the finite lattice model. Their result, $\alpha=0.42 \pm 0.03$ is presently considered to be quite reliable.Happily, it agrees closely with the value found for the helium monolayer ordering transition! Further theoretical treatments incorporating finite wells, high mobility and size effects should bring these models closer to the physical system.

Finally, the phase diagram of ordered helium is also important. The existing data near the critical density /13/ was fitted we11 by a recent position-space renormalization-group treatment using the interparticle interaction as the only adjustable parameter /19/. Further experimentation for comparison over the whole phase diagram is definitely warrented. Careful probing should be done of the two-phase regions on either side of the $\sqrt{3}$ phase. The low density $2 D$ liquid somehow merges with the ordered phase and the ordered solid might coexist with the close-pack high density solid. Sharp specific heat peaks near $1 \mathrm{~K}$ have already been noted here $/ 21 \%$. Researchers are currently seeking a good 4-state Potts transition which, according to Baxter and Wu $/ 20 /$, should be very strong with $\alpha=2 / 3$. Helium films in the region between liquid and $\sqrt{3}$ ordered phase (see figure 1) might be trying to form a $(2 \times 2)$ array $/ 10 /$. So far, no dramatic structure has been found, but investigations using more uniform substrates than Grafoil should yield more definitive results. 
References

/1/ Marseille Conf. on Physisorption, J. de Phys. 38 (1977) Supp1. $\mathrm{N}^{\circ} 10$

/2/ Domany, E., and Riede1, E.K., Phys. Rev. Lett. 40 (1978) 561

/3/ Thomy, A., and Duva1, X., J. Chim. Phys. 67 (1970) 1101

14/ See Dash, J.G., Films on Solid Surfaces (Academic Press) 1975

/5/ Potts, R.B., Proc. Camb. Phil. Soc. 48 (1952) 106

/6/ Alexander, S., Phys. Lett. 54A (1975) 353

17/ Larher, Y., This Conference

/8/ Domany, E., Schick, M., and Walker, J.S., Phys. Rev. Lett. 386 (1977) 1148

19/ Novaco, A.D., Private Communication

/10/ Bretz, M., Dash, J.G., Hickerne11, D.C., McLean, E.0., and Vilches, O.E., Phys. Rev. A8 (1973) 1589

/11/ Grafoil and ZYX are exfoliated graphite products of Union Carbide Corp.

/12/ Dash, J.G., Phys. Reports 38 (1978) 177

/13/ Bretz, M., Phys . Rev. Lett. 38 (1977) 501

/14/ Straley, J.P., and Fisher, M.E., J. Phys. A6 (1973) 1310

/15/ Baxter, R.J., J. Phys. C6 (1973) L445

/16/ Kin, D., and Joseph, R.I., J. Phys. A8 (1975) 891 ; Zwanzig, R., and Ramshaw, J.D., J. Phys. Alo (1977) 65

/17/ Berker, A.N., and Wortis, M., Phys. Rev. B14 (1976) 4946 ; Burkhardt, T.W., Knops, H.J.F., and Den Ni $\overline{j s, ~ M ., ~ J . ~ P h y s . ~}$ A9 (1976) L179 ; Dasgupta, C., Phys. Rev. B15 (1977) 3460

/18/ De Neef, T., and Enting, I.G., J. Phys. Al0 (1977) 801

/19/ Schick, M., Walker, J.S., and Wortis, M., Phys, Rev, B16 (1977) 2205

/20/ Baxter, R.J., and Wu, F.Y., Phys. Rev. Lett. 31 (1973) 1294

/21/ Hering, S., Van Sciver, S.W., and Vilches, O.E., J. Low Temp. Phys. 25 (1976) 793 